\title{
Patient Volume and Dispositions in a VA Psychiatric Emergency Room During COVID-19
}

\author{
Lorena Mitchell $^{1} \cdot$ Brian Fuehrlein $^{1}$ (I)
}

Received: 16 October 2020 / Accepted: 11 January 2021 / Published online: 30 January 2021

(c) This is a U.S. government work and not under copyright protection in the U.S.; foreign copyright protection may apply 2021

\begin{abstract}
The COVID-19 pandemic led to a decline in emergency department visits in the United States. Less is known about how COVID-19 has impacted psychiatric emergency services (PES). This report compares visits to the Connecticut Department of Veterans Affairs psychiatric emergency room from March-August 2020 to the same period from three prior years (2017, $2018,2019)$ to evaluate the impact of COVID-19 on patient volume and dispositions. Compared to prior years, there was a decline in visits in March-August 2020, particularly in April. This coincided with the peak of COVID-19 in the state. Rates of hospitalizations remained consistent, while rates of referrals to residential programs decreased. The decline in visits likely indicates that many veterans postponed necessary mental health and substance use treatment. This delay could have significant clinical ramifications for veterans and may lead to an eventual surge in demand for emergency mental health care.
\end{abstract}

Keywords COVID-19 $\cdot$ Veterans $\cdot$ Emergency $\cdot$ Mental health $\cdot$ Patient volume

\section{Introduction}

The COVID-19 pandemic has led to a sharp decline in emergency department (ED) visits in the United States and worldwide. One CDC study found a $42 \%$ decline in ED visits during the early weeks of the pandemic compared to the same period a year earlier (Hartnett et al. 2020).

Less is known about how COVID-19 has impacted usage of psychiatric emergency services (PES). With increased unemployment, altered outpatient mental health services, reduced community resources, i.e. drop-in centers and shelters, and increased social isolation, there are reasons to believe that more people may require acute psychiatric services. However, at least one recent review suggests that PES also experienced a decline in visits during the first months of the COVID-19 outbreak. Yale New Haven Hospital's PES volume decreased approximately $26 \%$ during the peak of the COVD-19 outbreak in Connecticut (Goldenberg and Parwani 2020).

Brian Fuehrlein

Brian.fuehrlein@va.gov

1 Department of Veterans Affairs, Yale University School of Medicine, 950 Campbell Ave, West Haven, CT 06516, USA
A few miles from Yale New Haven Hospital, the Department of Veteran's Affairs (VA) in Connecticut provides 24-h services to veterans with any psychiatric or substancerelated emergency in a 12-bed secured emergency room unit that serves veterans across the state. Common reasons for presenting to the ER and receiving PES include substance use, suicidal or homicidal ideation, PTSD, psychosis, and other psychosocial crises. Not only does VA PES triage and stabilize psychiatric emergencies, it also acts as an important resource hub by referring and transferring veterans directly to residential treatment programs, connecting veterans to housing resources, and coordinating outpatient mental health and other health services. The VA tends to serve a vulnerable subset of veterans who are more likely to be low income, belong to a minority group, be unemployed, and have a mental health disorder diagnosis (Agha et al. 2000; Eibner et al. 2016). In FY 2019, VA PES served 743 unique veterans with 1238 visits.

The first confirmed case of the novel coronavirus in the state occurred on March 8, 2020 (CT Department of Public Health 2020). On March 16, Governor Lamont closed schools, suspended non-essential businesses, and instructed residents to stay home effective March 23 (State of Connecticut 2020). COVID-19 cases peaked with 1,179 new cases confirmed on April 14 (CT Department of Public Health 2020). The state began a multi-phase reopening on 
May 20 continuing through September. Given this timeline of COVID-19 in the state and spurred by Goldenberg and Parwani's findings, our study analyzes how COVID-19 impacted VA Connecticut's PES volume by comparing the number of visits between March-August 2020 to the same period from the three prior years $(2017,2018,2019)$. We consider reasons for usage decline and identify gaps in services created by the public health crisis.

\section{Methods}

For administrative and tracking purposes, basic information on all patients who present to VA PES is collected and logged. These data include demographic information, psychiatric diagnosis, presenting complaint, and disposition plan. De-identified data for the relevant time periods were analyzed.

Rates of hospitalizations were calculated based on the number of veterans who were admitted to an inpatient psychiatric or detoxification unit. VA PES transfers veterans to its own inpatient unit as well as non-VA community hospital inpatient units. Rates of referrals to residential rehabilitation programs comprises veterans who were referred to either a VA partial hospitalization program, a VA crisis respite program, or a non-VA 28-day residential program.

This study was approved by the VA Connecticut Healthcare System Human Research Protection Program. The authors certify responsibility for this report.

\section{Results}

The overall mean monthly presentations to VA PES in March-Aug 2017-2019 was 114 (122 in 2017, 113 in 2018 and 107 in 2019). In March-August 2020 the mean decreased to 82 (77 in March, 60 in April, 89 in May, 86 in June, 100 in July, and 82 in August). Compared to 2017-2019, in 2020 there was a 31.9\% decrease in March, 49.4\% decrease in April (corresponding to the peak of COVID-19 in Connecticut), 23.5\% decrease in May, 21.6\% decrease in June, 9.9\% decrease in July, and 29.5\% decrease in August.

In March-August 2017-2019 the overall mean rate of admissions was $49.3 \%$ and in 2020 it was $48.8 \%$. The consistency in admission rates despite the volume decrease suggests that patient acuity was similar to previous years.

In March-August 2017-2019 the overall mean rate of referrals to residential rehabilitation programs was $8.2 \%$ or 9 veterans per month. In March-August 2020, the mean rate of referrals was 6.3\%. In April and May 2020, mean rate of referrals to residential treatment was $3.4 \%$, the lowest observed percentages during the study's time period with only two veterans referred in April and three veterans referred in May. Referrals to residential programs began to increase in June, reaching pre-COVID-19 levels by July.

\section{Discussion}

Compared to prior years, the number of visits to VA Connecticut PES declined in March-August of 2020. Many veterans likely postponed necessary emergency mental health and substance use care. In fact, because VA PES serves as a safety net for particularly vulnerable veterans by providing referrals to mental health services, housing, residential programming, and other social services, veterans who avoided visiting the hospital and lacked access to other outpatient services may have been disproportionally impacted by their delaying of care.

The overall decline in patient volume is likely due to a confluence of factors. As documented at hospitals across the country by the American College of Emergency Physicians (2020), patients reported avoiding seeking emergency care due to fears of contracting COVID-19. In addition, veterans may have incorrectly believed that the hospital was closed or that emergency mental health services were suspended, especially early in the pandemic when many medical resources were unavailable.

Reduced PES volume may also be attributed to an increased use of tele-mental health services and increased veteran outreach. VA Connecticut migrated all outpatient mental health contact to telephone or video appointments on March 20, 2020 with increased telephone outreach to veterans flagged as high risk for suicide. Nationally, in March 2020 the Veteran's Crisis Line experienced an 11\% increase in calls and online chats, and a 7\% increase texts from the previous month (Department of Veterans Affairs-Office of Inspector General 2020), suggesting that veterans were experiencing more distress during this time and/or had less access to usual resources. However, in Connecticut there was no increase in locally placed calls to the Veterans Crisis Line: 43 calls in March and 40 calls in April 2020, compared to 44 and 42 respectively in 2019. There was a slight increase in May 2020 with 63 calls, compared to 46 the year prior. This may support the idea that veterans in the state were being well-served by non-emergency tele-mental health outreach and appointments. However, given the vulnerable subset of veterans served by VA PES, i.e., lack of access to technology or not well engaged in outpatient treatment, it is likely that a number of veterans were receiving neither PES services nor outpatient tele-mental health care.

Compared to prior years, rates of hospitalizations remained consistent throughout the pandemic, indicating that the acuity of those who presented likely remained similar. Given the overall decline in visits with consistent rates 
of hospitalizations, it is likely that many veterans requiring hospitalization opted not to present to the VA and receive emergency psychiatric care. It is reasonable to believe that veterans who delayed care will eventually present to the emergency room. There is also reason to believe there will be an increase in demand for PES given the ongoing stress caused by the pandemic (unemployment, social isolation, etc.). Finally, the loss of traditional services may weigh heavily on a vulnerable population served by PES. Thus, the question remains whether there will be a significant increase in PES patient volume in the coming months and whether the system is designed to handle such a surge.

The decline in transfers to residential programs is an example of an urgent service gap as a result of the pandemic. VA PES acts as a gateway to residential rehabilitation programs. VA PES witnessed a decline in these referrals, especially in April and May. On March 16, 2020, the VA's on-site 21-day residential program closed due to COVID19 precautions. The loss of this primary VA service, along with others, created challenges in finding timely and appropriate residential rehabilitation programming for veterans. It is possible that the overall decline in PES volume was related to reduced residential programming as veterans may have learned that this resource was no longer available and deferred presenting to VA PES. Referral rates increased as programs began to reopen.

This study is limited in scope due to the recency of the COVID-19 pandemic. We can only speculate on the factors that drive a patient to seek (or not seek) psychiatric emergency care during a pandemic and how these factors relate to those choosing to seek medical ED care. As more time elapses and more data are available, research about veteran demographics (age, race, gender, housing status) and diagnostic categories (substance use, mood disorders, psychosis) may reveal more about the patterns of care-seeking during this global pandemic.

Funding No funding was received to assist with the preparation of this manuscript.

\section{Compliance with ethical standards}

Conflict of interest The authors have no conflicts of interest to declare that are relevant to the content of this article.
Ethical Approval This study was approved by the VA Connecticut Healthcare System Human Research Protection Program.

\section{References}

Agha, Z., Lofgren, R. P., VanRuiswyk, J. V., \& Layde, P. M. (2000). Are Patients at Veterans Affairs Medical Centers Sicker? A Comparative Analysis of Health Status and Medical Resource Use. Archives of Internal Medicine, 160(21), 3252-3257. https://doi. org/10.1001/archinte.160.21.3252.

American College of Emergency Physicians. (2020). Public poll: Emergency Care Concerns Amidst Covid-19. https://www.emergencyp hysicians.org/globalassets/emphysicians/all-pdfs/acep-mc-covid 19-april-poll-analysis.pdf.

CT Department of Public Health. (2020). COVID-19 Data Summary. [Data Set]. Retrieved from https://data.ct.gov/stories/s/COVID -19-data

Department of Veterans Affairs, Office of Inspector General. (2020). Veterans Crisis Line Challenges, Contingency Plans, and Successes During the COVID-19 Pandemic. (Report \#20-02830-05). Retrieved from https://www.va.gov/oig/pubs/VAOIG-20-02830 -05.pdf

Eibner, C., Krull, H., Brown, K., Cefalu, M., et al. (2016). Current and Projected Characteristics and Unique Health Care Needs of the Patient Population Served by the Department of Veterans Affairs. Rand Health Q., 5(4), 13.

Goldenberg, M. N., \& Parwani, V. (2020). Psychiatric emergency department volume during Covid-19 pandemic. American Journal of Emergency Medicine, S0735-6757(20), 30450-30452. https:// doi.org/10.1016/j.ajem.2020.05.088.

Hartnett, K. P., Kite-Powell, A., DeVies, J., et al. (2020). Impact of the COVID-19 Pandemic on Emergency Department Visits - United States, January 1, 2019-May 30, 2020. MMWR, 69(23), 699-704. https://doi.org/10.15585/mmwr.mm6923e1.

State of Connecticut, Office of the Governor. (2020, March 20). Governor Lamont Signs Executive Order Asking Connecticut Businesses and Residents: 'Stay Safe, Stay Home'. [Press Release]. Retrieved from https://portal.ct.gov/Office-of-the-Governor/News/ Press-Releases/2020/03-2020/Governor-Lamont-Signs-ExecutiveOrder-Asking-Connecticut-Businesses-and-Residents-Stay-Safe

Publisher's Note Springer Nature remains neutral with regard to jurisdictional claims in published maps and institutional affiliations. 